\title{
Anterior Skull Base Glomangioma-Induced Osteomalacia
}

\author{
Malia S. Gresham ${ }^{1}$ Steven Shen ${ }^{2} \quad$ Yi J. Zhang ${ }^{3} \quad$ Kelly Gallagher ${ }^{4}$
}

\footnotetext{
${ }^{1}$ Department of Otolaryngology-Head and Neck Surgery, Baylor College of Medicine, Houston, Texas, United States

2 Department of Pathology and Genomic Medicine, Houston Methodist Hospital, Houston, Texas, United States

${ }^{3}$ Division of Neurological Surgery, Department of Neurosurgery, Houston Methodist Hospital, Houston, Texas, United States

${ }^{4}$ Department of Otolaryngology-Head and Neck Surgery, Baylor College of Medicine, Houston, Texas, United States
}

\begin{abstract}
Address for correspondence Malia S. Gresham, BA, Baylor College of Medicine, 8100 Cambridge St. \#85, Houston, TX 77054, United States (e-mail: maliagresham@gmail.com).
\end{abstract}

J Neurol Surg Rep 2017;78:e9-e11.

\begin{abstract}
Keywords

- oncogenic osteomalacia

- glomangioma

- paranasal sinus tumor

- glomus tumor

Oncogenic osteomalacia $(\mathrm{OO})$ is an uncommon but treatable cause of osteomalacia related to tumor production of FGF23, usually caused by benign mesenchymal neoplasms. Paranasal sinus glomangiomas are a rare cause of $\mathrm{OO}$, with only one previously reported case. Here we describe a second case (first reported in English) of paranasal sinus glomangioma-induced osteomalacia in a 42-year-old man. He presented with weakness and multiple spontaneous fractures, and was found to have an ethmoid sinus glomangioma with intracranial extension. The tumor was removed via endoscopic endonasal approach to the anterior skull base, which resulted in complete resolution of symptoms and no further evidence of disease 1 year postoperatively.
\end{abstract}

\section{Introduction}

A glomangioma is a benign vascular tumor derived from the glomus body, a specialized neuromyoarterial structure involved in thermal regulation. It is a subtype of the more generalized category of "glomus tumors" and should not be confused with the head and neck paragangliomas, such as the glomus tympanicum or glomus jugulare. It should also be distinguished from the glomangiopericytoma, the sinonasal type hemangiopericytoma, which displays different ultrastructural and histochemical characteristics. ${ }^{1}$ Glomangiomas are most commonly found subungually and are exceedingly rare in the head and neck. ${ }^{2}$ They account for only $0.6 \%$ of all nonepithelial tumors of the nasal cavity, nasopharynx, and paranasal sinuses. ${ }^{3}$ Glomangiomas that induce paraneoplastic osteomalacia are even more uncommon, with only one case reported to date. ${ }^{4}$ While there are several documented cases of oncogenic osteomalacia (OO) caused by glomangiopericytomas, ${ }^{5}$ we present the second reported case of glomangioma-induced osteomalacia and the first case documented in English.

\section{Case Report}

A 42-year-old man with a history of unexplained hip, rib, scapula, and metatarsal fractures; left foot pain with exercise; and weakness presented to our clinic for evaluation of a paranasal sinus tumor. The endocrinology service initially diagnosed him with tumor-induced osteomalacia caused by fibroblast growth factor 23 (FGF23), which prompted an octreotide scan revealing a questionable area of enhancement in the pituitary. A subsequent magnetic resonance imaging (MRI) showed an ethmoid mass extending through the cribriform intracranially, measuring $3.9 \times 1.9 \times 2.4 \mathrm{~cm}$ at its largest dimensions ( $\mathbf{- F i g .} \mathbf{1}$ ). The patient reported occasional sinus congestion, decreased sense of smell during the preceding 2 to 3 years and occasional blurry vision in the morning. He denied epistaxis, facial pressure or pain, or double vision. His laboratory workup revealed a significantly decreased 1,25-vitamin $D$ level of less than $8 \mathrm{ng} / \mathrm{mL}$, a decreased phosphate level to $1.6 \mathrm{mg} / \mathrm{dL}$, a low to normal calcium level of $8.7 \mathrm{mg} / \mathrm{dL}$, and a significantly elevated received

June 30, 2016

accepted after revision

November 7, 2016
DOI http://dx.doi.org/

10.1055/s-0036-1597599.

ISSN 2193-6358. (c) 2017 Georg Thieme Verlag KG Stuttgart · New York
License terms

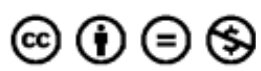




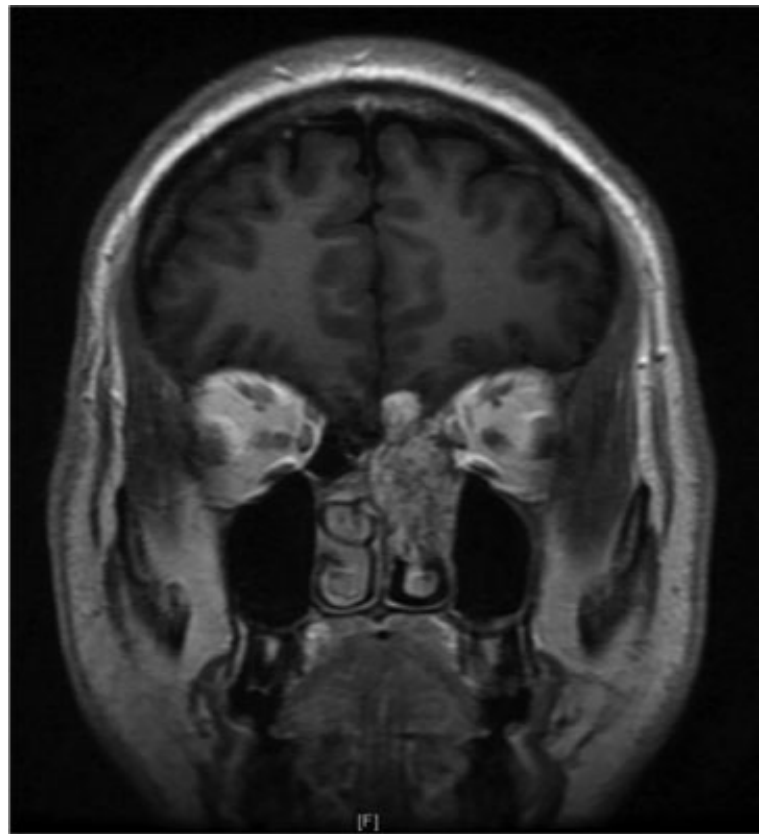

Fig. 1 Preoperative MRI, T1 with contrast, showing an ethmoid mass extending through the cribriform intracranially. Dimensions $3.9 \times 1.9 \times 2.4 \mathrm{~cm}$.

alkaline phosphatase level at $65.3 \mu \mathrm{g} / \mathrm{L}$. Rigid nasal endoscopy in clinic was unremarkable.

The patient underwent a combined endoscopic endonasal approach to the anterior skull base with tumor resection. The cribriform defect and intraoperative cerebrospinal fluid (CSF) leak were repaired with a pedicled nasoseptal flap. Pathology revealed a vascular neoplasm with a uniform cluster of ovoid cells arranged around the vessels and moderate focal nuclear enlargement (-Fig. 2). No necrosis or mitotic figures were seen. Tumor cells stained positive for CD31 and smooth

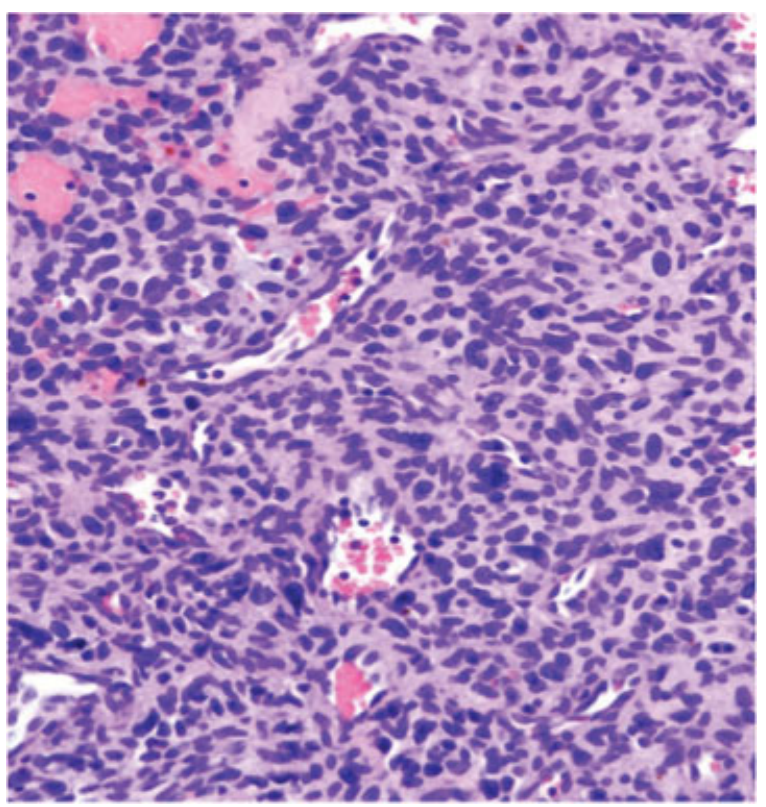

Fig. 2 Uniform clusters of ovoid cells arranged around vessels with moderate nuclear enlargement. H\&E, 200x.

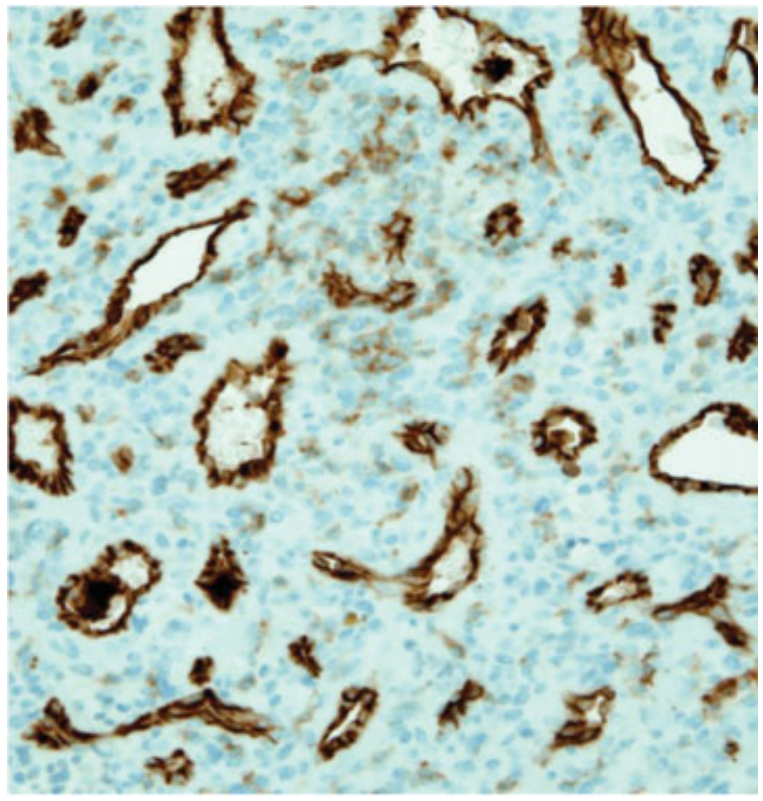

Fig. 3 Tumor cells stained positive for CD31. 200x.

muscle actin (SMA) but negative for CD34, S100, and pancytokeratin on immunohistochemistry (-Fig. 3). These characteristics are consistent with and provided the final diagnosis of glomangioma.

At 1 year follow-up, the patient reported near-complete resolution of bone pain and improvement in smell, and he had normalization of phosphate, alkaline phosphatase, vitamin D, and other laboratory values. Repeat MRI showed gross total resection of the mass and no detectable recurrence (-Fig. 4).

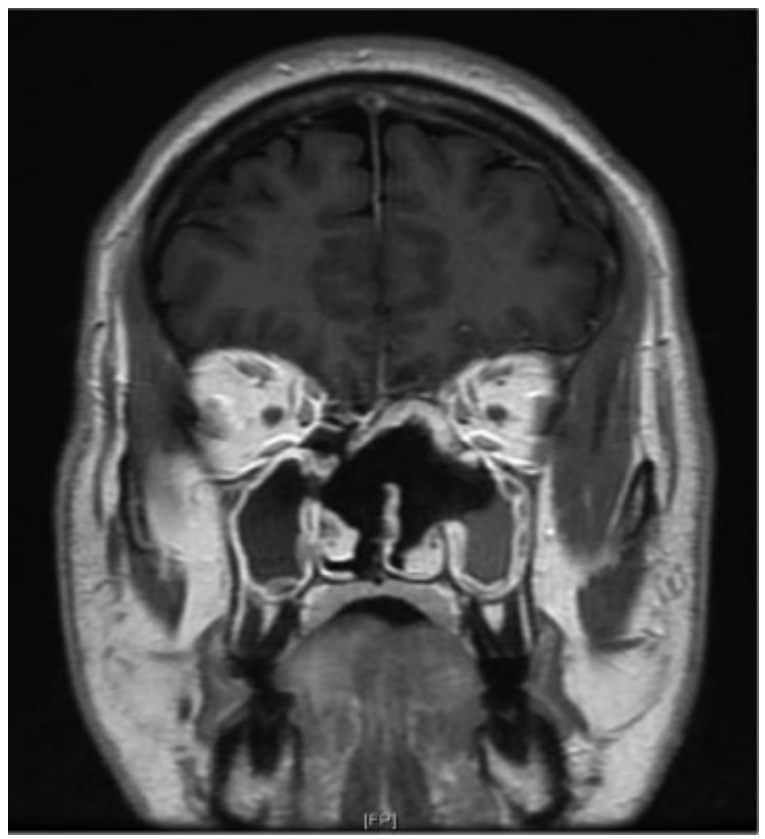

Fig. 4 Postoperative MRI, T1 with contrast, showing gross total resection of the mass with nasoseptal flap reconstruction of the skull base. 


\section{Discussion}

This patient suffered from 00 for several years due to undiagnosed ethmoid glomangioma. Osteomalacia is a disease of the bone characterized by defective mineralization of osteoid from decreased levels of available phosphate and calcium or increased bone resorption. It often presents with diffuse joint and bone pain, easy fracturing, difficulty walking, weakness, and other nonspecific symptoms.

$\mathrm{OO}$ is a rare, disabling, and curable form of osteomalacia that affects both sexes equally and usually presents around 40 years of age. ${ }^{6}$ It is not well described in glomangiomas but has been detailed several times in relation to glomangiopericytomas and other soft tissue and bone tumors, with more than 300 reported cases ${ }^{7}$ since its debut in $1947 .{ }^{8}$ It predominantly occurs in the context of mesenchymal tumors and is thought to be due to neoplastic overexpression of FGF23. This protein inactivates the sodium-phosphate pump in the proximal tubule (prohibiting phosphate reabsorption and inducing renal phosphate wasting) and reduces 1-hydroxylation of 25-hydroxy vitamin D. ${ }^{9}$ Accordingly, common OO laboratory abnormalities include hypophosphatemia, normal or decreased calcium, decreased 1,25-dihydroxy vitamin D3 with resistance to vitamin D supplementation, and elevated alkaline phosphatase, which our patient exhibited.

Tumors causing $\mathrm{OO}$ tend to be small, occult, and slow growing, making diagnosis remarkably difficult. When other causes of osteomalacia have been ruled out and $\mathrm{OO}$ is suspected, clinicians should consider measuring a serum FGF23 level. Elevated FGF23 in this setting should then prompt full body imaging (including hands and feet) to expose the lesion. The current imaging standard for investigation of $\mathrm{OO}$ is whole-body MRI short tau inversion recovery (STIR). ${ }^{10}$ Another commonly used modality is octreotide scintigraphy (Octreoscan), which utilizes a radiotracer that binds to somatostatin receptors overexpressed on the causative tumor. ${ }^{11}$ Once the neoplasm is localized, surgical resection with wide margins is the definitive treatment. It is curative in essentially all cases and typically leads to rapid normalization of laboratory values and reversal of most clinical symptoms.
The nonspecific presentation of osteomalacia, the obscure nature of the tumors that cause $\mathrm{OO}$, and the rarity of glomangiomas in the head and neck make this patient's diagnosis challenging. We believe this to be the second reported case of paranasal sinus glomangioma-induced osteomalacia and the first reported in English. Many head and neck surgeons may not be aware of its existence or consider it in their differential diagnoses of sinus tumors. This report serves to increase the awareness of this uncommon pathology so that it may be considered and treated in future cases.

\section{References}

1 Constantinidis J, Kiefer A, Reitnauer K, Iro H. Glomangioma of the nasal cavity and paranasal sinuses. Rhinology 2000;38(3):136-139

2 Tsuneyoshi M, Enjoji M. Glomus tumor: a clinicopathologic and electron microscopic study. Cancer 1982;50(8):1601-1607

$3 \mathrm{Fu}$ YS, Perzin KH. Non-epithelial tumors of the nasal cavity, paranasal sinuses, and nasopharynx: a clinicopathologic study. I. General features and vascular tumors. Cancer 1974;33(5): 1275-1288

4 Wang T, Qu S, Ye L. [Sinonasal glomus tumour-induced osteomalacia: a case report] [in Chinese]. Zhonghua Er Bi Yan Hou Tou Jing Wai Ke Za Zhi 2014;49(8):691-692

5 Beech TJ, Rokade A, Gittoes N, Johnson AP. A haemangiopericytoma of the ethmoid sinus causing oncogenic osteomalacia: a case report and review of the literature. Int J Oral Maxillofac Surg 2007; 36(10):956-958

6 Gonzalez-Compta X, Mañós-Pujol M, Foglia-Fernandez M, et al. Oncogenic osteomalacia: case report and review of head and neck associated tumours. J Laryngol Otol 1998;112(4):389-392

7 Folpe AL, Fanburg-Smith JC, Billings SD, et al. Most osteomalaciaassociated mesenchymal tumors are a single histopathologic entity: an analysis of 32 cases and a comprehensive review of the literature. Am J Surg Pathol 2004;28(1):1-30

8 McCANCE RA. Osteomalacia with Looser's nodes (Milkman's syndrome) due to a raised resistance to vitamin $\mathrm{D}$ acquired about the age of 15 years. Q J Med 1947;16(1):33-46

9 Ray S, Chakraborty PP, Biswas K, Ghosh S, Mukhopadhyay S, Chowdhury S. A case of oncogenic osteomalacia due to occult nasal sinus tumor. Clin Cases Miner Bone Metab 2015;12(1):65-68

10 Sundaram M, McCarthy EF. Oncogenic osteomalacia. Skeletal Radiol 2000;29(3):117-124

11 Nguyen BD, Wang EA. Indium-111 pentetreotide scintigraphy of mesenchymal tumor with oncogenic osteomalacia. Clin Nucl Med 1999;24(2):130-131 